\title{
Cosmological Tests using Redshift Space Clustering in BOSS DR11
}

\author{
Yong-Seon Song ${ }^{1}$, Cristiano G. Sabiu ${ }^{2}$, Teppei Okumura ${ }^{3}$, Minji Oh ${ }^{1,4}$, Eric V. Linder ${ }^{1,5 *}$ \\ ${ }^{1}$ Korea Astronomy and Space Science Institute, Daejeon 305-348, Korea \\ ${ }^{2}$ Korea Institute for Advanced Study, Dongdaemun-gu, Seoul 130-722, Korea \\ ${ }^{3}$ Kavli Institute for the Physics and Mathematics of the Universe (Kavli IPMU, \\ WPI), The University of Tokyo, Chiba 277-8582, Japan \\ ${ }^{4}$ University of Science and Technology, Daejeon 305-333, Korea and \\ ${ }^{5}$ Berkeley Lab and Berkeley Center for Cosmological Physics, \\ University of California, Berkeley, CA 94720, USA
}

(Dated: September 10, 2021)

\begin{abstract}
We analyze the clustering of large scale structure in the Universe in a model independent method, accounting for anisotropic effects along and transverse to the line of sight. The Baryon Oscillation Spectroscopy Survey Data Release 11 provides a large sample of 690,000 galaxies, allowing determination of the Hubble expansion $H$, angular distance $D_{A}$, and growth rate $G_{\Theta}$ at an effective redshift of $z=0.57$. After careful bias and convergence studies of the effects from small scale clustering, we find that cutting transverse separations below $40 \mathrm{Mpc} / h$ delivers robust results while smaller scale data leads to a bias due to unmodelled nonlinear and velocity effects. The converged results are in agreement with concordance $\Lambda$ CDM cosmology, general relativity, and minimal neutrino mass, all within the $68 \%$ confidence level. We also present results separately for the northern and southern hemisphere sky, finding a slight tension in the growth rate - potentially a signature of anisotropic stress, or just covariance with small scale velocities - but within $68 \%$ CL.
\end{abstract}

PACS numbers: 98.80.-k,95.36.+x

\section{INTRODUCTION}

Three dimensional maps of galaxy positions over wide sky areas are greatly advancing our cosmological knowledge. The clustering of galaxies measures the growth of large scale structure and echoes the baryon-photon sound horizon scale in the extra power of the baryon acoustic oscillation feature. Studying clustering along the line of sight (in the redshift direction) and transverse to the line of sight (in the angular direction) probes the Hubble expansion and the angular diameter distance respectively [1-4]. Such anisotropic effects have been extensively analyzed in various redshift surveys [e.g., 510]. Combining the radial and transverse information can measure the cosmically induced shear of the clustering, known as the Alcock-Paczynski effect [11, 12].

Here we use the 690,000 galaxies of the Baryon Oscillation Spectroscopic Survey (BOSS) Data Release 11 (DR11) to carry out a model independent anisotropic clustering analysis, measuring the Hubble parameter $H$, angular distance $D_{A}$, and growth rate variable $G_{\Theta}$ in a volume with effective redshift $z_{\text {eff }}=0.57$. This analysis does not assume any specific dark energy model or even the Friedmann-Robertson-Walker relation between the expansion rate $H$ and distance $D_{A}$, nor the general relativity relation between expansion and growth $G_{\Theta}$ [13].

This work closely follows our approach $[13,14]$ with BOSS DR9 simulation and data, with several improvements arising from both the BOSS data (see [14]) and our analysis. The data covers a wider sky area, much

\footnotetext{
*ysong@kasi.re.kr
}

more uniformly, and the computational simulations for the mock catalogs take into account more instrumental effects. In our analysis the improved data quality allows straightforward use of the covariance matrix without need for the previous singular value decomposition to control noise. We also study in more detail the dependence of the results on the small scale clustering and their convergence behavior as this regime, with uncertain nonlinearity and velocity effects, is truncated.

Section II summarizes briefly our approach to fitting the clustering correlation function, concentrating on differences from $[13,14]$. We measure the anisotropic clustering in Section III. In Section IV we present the joint likelihood results for the cosmological quantities of the expansion $H$, distance $D_{A}$, and growth $G_{\Theta}$, and assess consistency with the concordance cosmology, neutrino mass, and general relativity. We also investigate the dependence of the cosmological results on the small scale cut-off, in terms of both bias and precision, and explore the comparison of northern and southern Galactic hemisphere data. We summarize and conclude in Section V.

\section{THEORETICAL MODEL AND SIMULATION}

\section{A. Theoretical model}

The observed galaxy two-point correlation function, $\xi$, is given by

$$
\xi(\sigma, \pi)=\int \frac{d^{3} k}{(2 \pi)^{3}} P(k, \mu) e^{i \mathbf{k} \cdot \mathbf{s}},
$$

where $\sigma$ and $\pi$ are the separations between the galaxies of the pair in the transverse and radial directions respec- 
tively, with respect to the observer, and $s$ is the total separation $s=\left(\sigma^{2}+\pi^{2}\right)^{1 / 2}$. The correlation function is the Fourier transform of the power spectrum $P$, with $\vec{k}$ the wavemode and $\mu$ the cosine of the angle between $\vec{k}$ and the line of sight.

The two point correlation function $\xi$ as observed in redshift space (i.e. where the radial dimension is not true separation but distance as measured through redshift, involving both separation and radial velocity) involves two distinct effects from redshift space distortions (RSD) $[15,16]$. In the linear regime, the density fluctuations and peculiar velocities are coherently evolved through the continuity equation. Thus the known correlation function in real space from the linear perturbation theory developed by gravitational instability is uniquely transformed into $\xi(\sigma, \pi)$ in redshift space. Gravitational infall squeezes the clustering pattern in redshift space along the line of sight (i.e. the $\pi$-direction), enhancing the correlation function by the Kaiser factor [15]. In the non-linear regime, however, the observed correlation function appears elongated along the line of sight due to the random virial velocities of galaxies, called the Finger of God effect (FoG) [17]. Because the FoG effect smears into even large scales, the linear theory Kaiser effect is not appropriate along the $\pi$ direction $[18,19]$.

A more accurate description of these RSD effects is required for BOSS clustering data. Improved models were tested using simulations in [13], and applied to DR9 data in [14]. These corrections are briefly reviewed below.

The mapping between real space and redshift space has significant correlations between the density and velocity fields. Although it extends into a infinite series of polynomials, a few leading modes are dominant near the linear regime. While the original linear theory includes terms up to $\mu^{4}$, terms up to $\mu^{6}$ are necessary in the improved models; higher order terms can be safely ignored in the quasi-linear regime [20].

The cross-correlation spectrum between density $\delta$ and velocity $\Theta$ is not independently measured, but rather estimated from the measured auto-correlations of $\delta$ and $\Theta$, based upon the assumption of a perfect cross-correlation coefficient (which is valid only in the linear theory). The deviation of spectra from the linear theory is perturbatively calculated using the resummed perturbation theory called RegPT $[21,22]$. When restricting analysis to the quasi-linear regime, the result is the non-linear portions of the power spectra are better separated from the linear spectra; for the latter the assumption of perfect cross-correlation between density and velocity fields can be applied.

However, the FoG effect remains non-perturbative in this model. The non-linear smearing effect is dominant at first order, and can be parameterised by a velocity dispersion $\sigma_{p}$. Our theoretical models (as all others) are broken at scales in which higher order terms become important, however. This motivates us to introduce the cut-off scales and consider them carefully. We use two cut-offs: $s_{\text {cut }}$ and $\sigma_{\text {cut }}$. The $s_{\text {cut }}$ accounts for the limit of theoretical description of RegPT, and the $\sigma_{\text {cut }}$ reflects the unknown contamination of the residual FoG effect.

\section{B. Methodology and simulation test}

The observed clustering as a function of the transverse and radial distances is related to the density and velocity growth functions, and the FoG parameter $\sigma_{p}$. Cosmological information is extracted from the density and velocity functions.

From the clustering $\xi(\sigma, \pi)$, measured in comoving distances, the transverse and radial distances scale linearly with $D_{A}$ and $H^{-1}$ respectively. The evolution of clustering occurs coherently for all scales in linear theory, and its initial shape (scale dependence) is determined in the early universe. Using early universe information from the cosmic microwave background we denote this as a Planck (or WMAP9) prior. All evolution in the amplitude after the last scattering epoch informs us about late time cosmology. We denote the normalized density and coherent motion (velocity) growth functions as $G_{b}$ and $G_{\Theta}$. This is a model independent analysis in the sense that we do not require, or use, any specific assumptions on energy density components such as dark energy or curvature; indeed we do not even have to assume the FriedmannRobertson-Walker relation of $D_{A}$ as an integral of $H^{-1}$. Note that $G_{b}=b G_{\delta}$, where $b$ is the galaxy bias and in linear theory $G_{\delta}=D$, where $D$ is the growth factor. Similarly $G_{\Theta}=d D / d \ln a$ is growth rate, and is proportional to the sometimes used combination $f \sigma_{8}$ [23], with $G_{\Theta}=f \sigma_{8}\left(D_{0} / \sigma_{8,0}\right)$ where 0 denotes the present. See [14] for more details.

The spectra of the density and the velocity fields are naturally expected to receive nonlinear corrections. One of these corrections comes from the random motion of galaxies, which results in the damping effect of the power spectrum amplitude. We apply a Gaussian FoG function with free parameter $\sigma_{p}$ characterizing the velocity. However, as these non-perturbative damping effects are not fully understood, we employ a cut-off scale to remove small scales where this is exacerbated, and study the effects of varying that scale.

In order to check the validity of our overall approach, we test it against simulations. We use the mock galaxy catalogs created by [24], which are designed to investigate the various systematics in the galaxy sample from Data Release 11 (DR11) of the Baryon Oscillation Spectroscopic Survey (BOSS) [25-27], referred to as the "CMASS" galaxy sample. In constructing the mock galaxy catalogs, [24] utilized second-order Lagrangian perturbation theory (2LPT) for the galaxy clustering driven by gravity, which enables the creation of a mock catalogs much faster than running an $N$-body simulation. The redshift range of galaxies in the catalog is $0.43<z<0.7$ and each catalog contains $\sim 7 \times 10^{5}$ galaxies, $90 \%$ of which are central galaxies residing in dark matter halos of $\sim 10^{13} h^{-1} M_{\odot}$. Table I illustrates that 


\begin{tabular}{lcc}
\hline \hline Parameters & Simulated values & Measured values \\
\hline$D_{A}\left(h^{-1} \mathrm{Mpc}\right)$ & 932.6 & $939.7_{-32.6}^{+26.7}$ \\
$H^{-1}\left(h^{-1} \mathrm{Mpc}\right)$ & 2177.5 & $2120.5_{-100.6}^{+82.3}$ \\
$G_{\Theta}$ & 0.46 & $0.47_{-0.07}^{+0.10}$ \\
\hline \hline
\end{tabular}

TABLE I. We demonstrate the recovery of input simulation values from our analysis pipeline using the $2 \mathrm{D}$ clustering model. The measured values of $D_{A}, H^{-1}$ and $G_{\Theta}$, and their $68 \%$ confidence level uncertainties for each realization, agree well with the input simulation values.

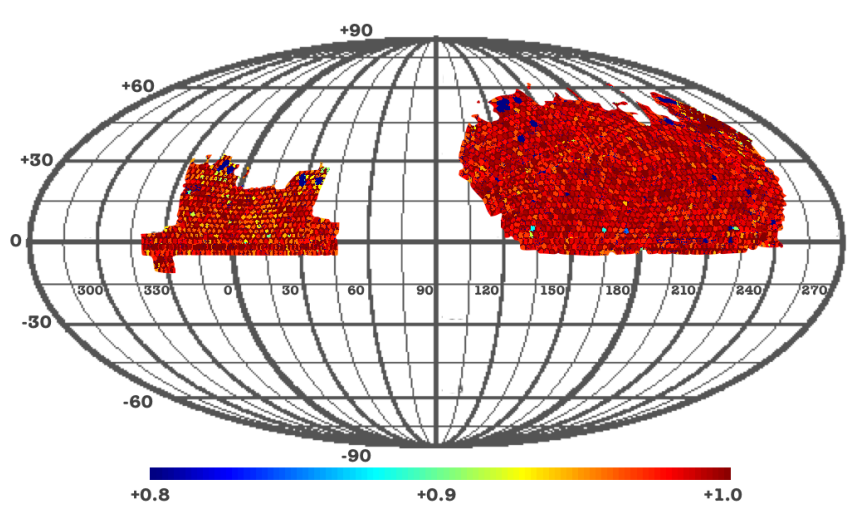

FIG. 1. The DR11 CMASS completeness map in Mollweide projection. The North and South patches are centred at RADec locations $(185,25)$ and $(2,10)$ respectively.

our analysis successfully recovers the simulated values for the cosmological quantities $D_{A}, H^{-1}$ and $G_{\Theta}$. Our studies show that $s_{\text {cut }}=50 h^{-1} \mathrm{Mpc}$ and $\sigma_{\text {cut }}=40 h^{-1} \mathrm{Mpc}$ give converged, robust results.

\section{MEASUREMENTS}

In our analysis we utilise the updated data release (DR11) of the Baryon Oscillation Spectroscopic Survey [BOSS; 28-30] which is part of the larger Sloan Digital Sky Survey [SDSS; 31, 32] program. From DR11 we focus our analysis on the Constant Stellar Mass Sample (CMASS) [33], which contains 690,826 galaxies and covers the redshift range $z=0.43-0.7$ over a sky area of $\sim 8,500$ square degrees. The angular coverage of DR11 CMASS is shown in Fig. 1. The majority of CMASS galaxies are bright, central galaxies (in the halo model framework) and are thus highly biased $(b \sim 2)$ [34].

Each spectroscopically observed galaxy is weighted to account for three distinct observational effects: redshift failure, $w_{\text {fail }}$; minimum variance, $w_{\mathrm{FKP}}[35]$; and angular variation, $w_{\text {sys }}$, which accounts for airmass dependent seeing effects and stellar contamination. These weights are described in more detail in [25] and [36]. The total weight for each galaxy is calculated as the product of these weights, i.e., $w_{\text {total }}=w_{\text {fail }} w_{\mathrm{FKP}} w_{\text {sys }}$. The random

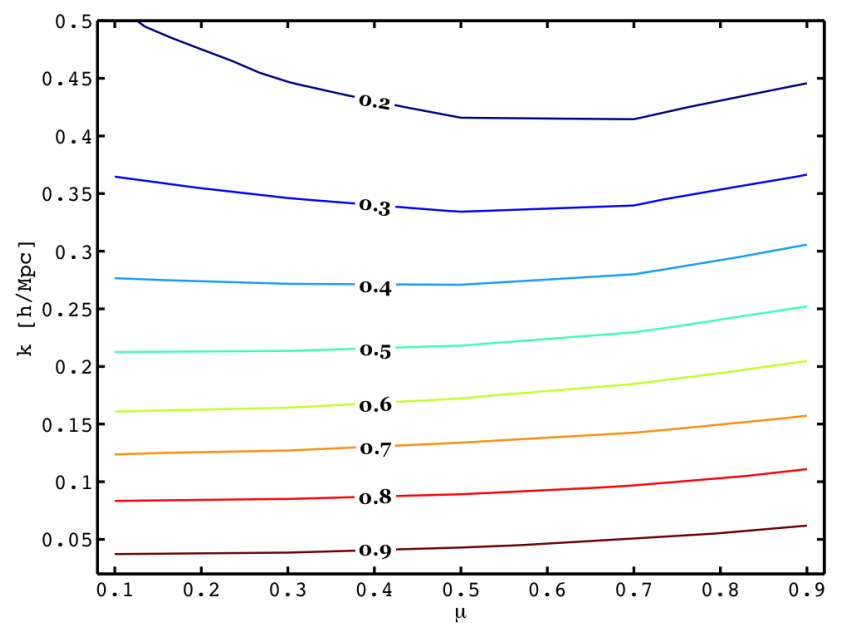

FIG. 2. The normalized effective volume $v(k, \mu)$, showing the sample variance completeness, is plotted. Values near unity mean that new volumes must be surveyed to gain further information on the density and velocity fields; lower values mean further galaxies can contribute information.

catalog points are also weighted but they only include the minimum variance FKP weight.

The CMASS galaxy sample is distributed over the range $0.43<z<0.7$, with an effective redshift of $z_{\text {eff }}=0.57$ and an effective volume of $V_{\text {eff }} \sim 6.0 \mathrm{Gpc}^{3}$ calculated as

$$
V_{\mathrm{eff}}=\sum\left(\frac{n\left(z_{i}\right) P_{0}}{1+n\left(z_{i}\right) P_{0}}\right)^{2} \Delta V\left(z_{i}\right),
$$

where $\Delta V(z)$ is the volume of a shell at redshift $z$ and $P_{0}=20,000 h^{-1} \mathrm{Mpc}$. To sample the density and velocity fields more fully, one can either increase the sampling density (raise $n P$ ) or survey new volumes. Figure 2 shows the sample variance completeness of the DR11 volume for each Fourier mode $k$ and $\mu$, i.e.

$$
v(k, \mu)=\left(\frac{\bar{n} P(k, \mu)}{1+\bar{n} P(k, \mu)}\right)^{2},
$$

where $\bar{n}$ is the average galaxy density within the volume, and the anisotropic power spectrum $P(k, \mu)$ is measured from mock simulations in [37]. When $v$ approaches unity (for high $n P$ ) then further information on the density field at that $k-\mu$ can only come from surveying different volumes; Fig. 2 can thus be thought of as showing the sample variance completeness.

We compute the redshift-space 2-dimensional correlation function $\xi(\sigma, \pi)$ of the BOSS DR11 galaxy catalog using the standard Landy-Szalay estimator [38]. In the computation of the correlation estimator we use a random point catalogue that constitutes an unclustered but observationally representative sample of the BOSS CMASS survey. The angular points are chosen to reside 


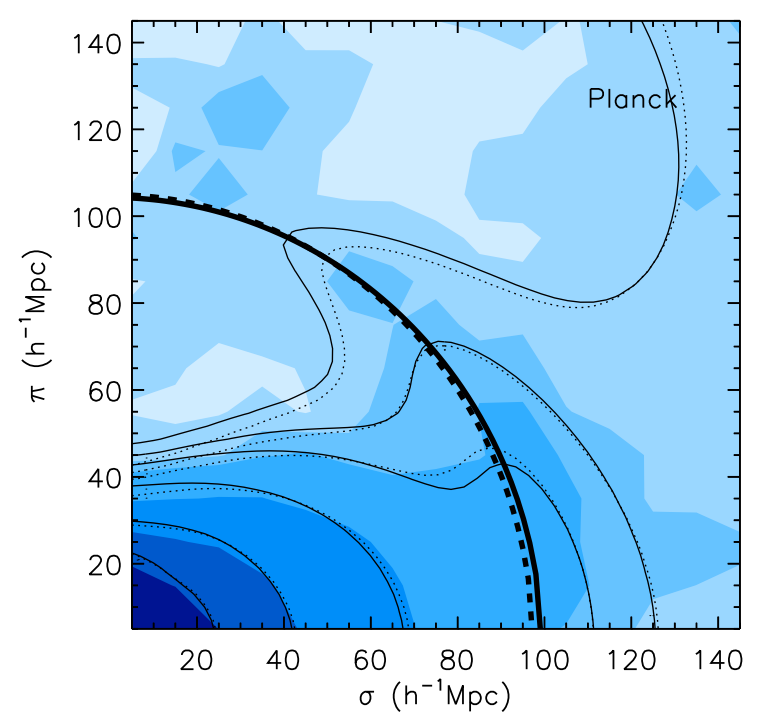

FIG. 3. The measured (blue filled contours), best fit (thin black), and LCDM-predicted (thin dotted) cases of $\xi(\sigma, \pi)$ are plotted, using the Planck early universe prior. The thick solid and dashed circles represent $2 \mathrm{D}$ BAO rings from the measurement and the Planck LCDM prediction.

within the survey geometry with a Monte Carlo acceptance proportional to the RA-Dec sector completeness, and the redshifts are obtained via the random shuffle method of [36]. The randoms are also assigned FKP weights, just as for the galaxies. To reduce the statistical variance of the estimator we use $\sim 50$ times as many randoms as we have galaxies. We perform the coordinate transforms using the fiducial Planck best fit flat $\Lambda \mathrm{CDM}$ cosmological model $\left(\omega_{b}=0.022068, \omega_{c}=0.12029, h=\right.$ $0.67)$.

We calculate the correlation function in 225 bins spaced by $10 h^{-1} \mathrm{Mpc}$ in the range $0<\sigma, \pi<$ $150 h^{-1} \mathrm{Mpc}$. The BOSS sample is naturally separated into North and South samples with 520,805 and 170,021 galaxies respectively, and we measure the anisotropic two-point correlation function in the north, south and combined samples.

Our results depend not only on the correlation functions but also on the errors of these measurements. Also, different bins of the correlation function can be strongly correlated to each other, therefore it is necessary to estimate a covariance matrix to give correct constraints on cosmological parameters.

For this purpose we use PTHALO [24] mock galaxy catalogs, updated to reflect the larger observational area. These catalogs were used for BAO analysis in [39] and have the same survey geometry and number density as the CMASS galaxy sample that was used in our analysis. The mocks catalogs constitute 600 density field realizations created using second-order Lagrangian per-

\begin{tabular}{c|cccc}
\hline \hline & Planck LCDM & Combined & North & South \\
\hline$D_{A}$ & 936.3 & $954.9_{-21.6}^{+19.8}$ & $955.1_{-21.7}^{+20.8}$ & $970.4_{-41.1}^{+35.8}$ \\
$H^{-1}$ & 2170.8 & $2159.8_{-117.8}^{+136.3}$ & $2207.2_{-124.0}^{+114.9}$ & $2220.9_{-358.0}^{+243.9}$ \\
$G_{b}$ & - & $1.15_{-0.08}^{+0.08}$ & $1.10_{-0.07}^{+0.07}$ & $1.15_{-0.19}^{+0.20}$ \\
$G_{\Theta}$ & 0.46 & $0.41_{-0.09}^{+0.09}$ & $0.34_{-0.08}^{+0.08}$ & $0.54_{-0.17}^{+0.16}$ \\
$\sigma_{p}$ & - & $6.2_{-3.8}^{+3.6}$ & $1.5^{+3.3}$ & $9.2_{-4.4}^{+3.4}$ \\
\hline \hline
\end{tabular}

TABLE II. We present the measured values of $D_{A}\left(h^{-1} \mathrm{Mpc}\right)$, $H^{-1}\left(h^{-1} \mathrm{Mpc}\right), G_{b}, G_{\Theta}$ and $\sigma_{p}\left(h \mathrm{Mpc}^{-1}\right)$, and their $68 \% \mathrm{CL}$ uncertainties, using the combined data, and the north and the south maps separately. Here the cutoffs are $s_{\text {cut }}=50 \mathrm{~h} \mathrm{Mpc}^{-1}$ and $\sigma_{\text {cut }}=40 \mathrm{hpc}^{-1}$.

turbation theory (2LPT). The density and velocity fields created using 2LPT eventually break down as one goes to small scales, but it was confirmed by $[6,24]$ that the correlation functions measured from the mock catalogs based on 2LPT match the one measured from the BOSS survey at scales larger than $20 h^{-1} \mathrm{Mpc}$. In our analysis below, we will use the data at $s \geq 50 h^{-1} \mathrm{Mpc}$ and, as we did in [13], to be conservative we will additionally remove the data along the line of sight, which is known to deviate from linear theory starting from larger scales than the data perpendicular to the line of sight (see Sec. IV below). Note that we use these mock catalogs solely to estimate errors of the correlation function measured from the CMASS sample.

For each realization we compute the correlation function as we did for the observed catalog and obtain a covariance matrix as described in detail in [14].

The measured two point correlation function $\xi(\sigma, \pi)$ is presented as blue filled contours in Fig. 3, with the levels of $(0.2,0.06,0.16,0.005,0.002,-0.001,-0.006)$ from the inner to outer contours. The RSD anisotropy is clearly visible, as is the $2 \mathrm{D} \mathrm{BAO}$ ring at $\sqrt{\sigma^{2}+\pi^{2}} \approx$ $100 h^{-1}$ Mpc.

\section{RESULTS}

\section{A. Cosmology from combined maps}

Information on the late-time cosmological expansion and dynamics are encoded in the distances along and transverse to the line of sight, and the growth of the density and velocity fields. A first consistency check of $D_{A}$ and $H^{-1}$ with the Planck LCDM model can be ascertained by comparing the two $2 \mathrm{D}$ BAO rings in Fig. 3 . The thick dashed and solid circles represent the rings estimated from the Planck LCDM model and the DR11 measurements. The growth functions do not alter the $2 \mathrm{D} \mathrm{BAO}$ ring, and it is distorted differently by $D_{A}$ and $H^{-1}$ in the transverse and radial directions. The consistency between the solid and dashed circles means that the measured distances agree well with the Planck LCDM model. 


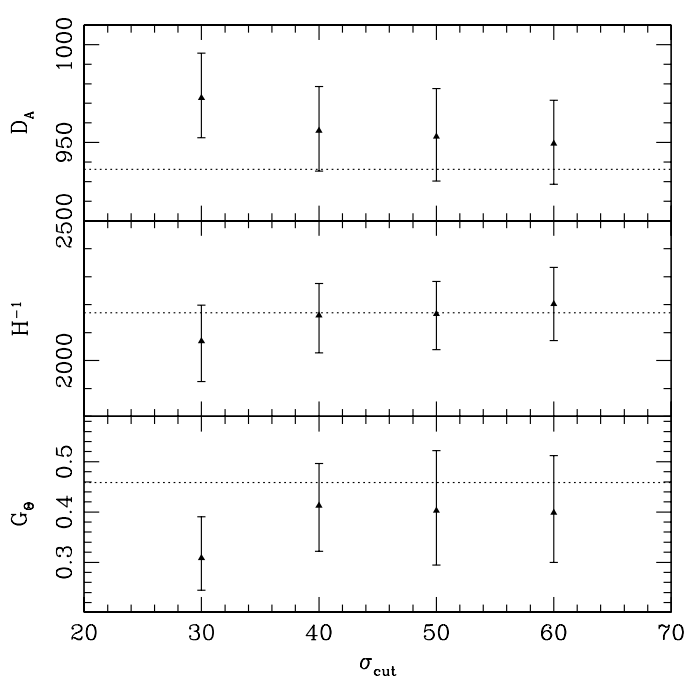

FIG. 4. The measured values of $D_{A}, H^{-1}$ and $G_{\Theta}$ are shown for various $\sigma_{\text {cut }}$ from $30 h \mathrm{Mpc}^{-1}$ and $60 h \mathrm{Mpc}^{-1}$. The values have converged for $\sigma_{\text {cut }} \geq 40 \mathrm{~h} \mathrm{Mpc}^{-1}$, but inclusion of smaller scales biases the answers by $\sim 1 \sigma$. The dotted lines, representing the Planck LCDM predictions, are shown purely for reference; the important aspect is convergence (not to any particular value).

We quantify this in Table II, where the measured $D_{A}$ and $H^{-1}$ are within the $68 \%$ confidence limit of the Planck LCDM prediction. We discuss the effect of $\sigma_{\text {cut }}$ below, and of North vs South maps in the next section.

For the density and velocity growth factors the information comes from multiple scales, and especially from the redshift space anisotropy. While the signal-tonoise of the inner (higher amplitude) contours of clustering is higher, the use of RegPT to second order is insufficient for accurate modelling of $\xi(\sigma, \pi)$ at scales $s<50 h \mathrm{Mpc}^{-1}$. In particular, the cross-spectrum between $\delta$ and $\Theta$ is not perfectly cross-correlated. When the cut-off $s<50 \mathrm{~h} \mathrm{Mpc}^{-1}$ is applied, the constraints on $G_{b}$ and $G_{\Theta}$ from their distinctive amplification of the inner contours [40] become weaker, but more robust as we now see.

At small scales, if the non-perturbative effect of FoG is underestimated, then the residual squeezing can be misinterpreted as a smaller $G_{\Theta}$. We expect the FoG effect to be increasingly important at smaller scales, and so these run increasing risk of misestimation. To test this, in Fig. 4 we show the cosmology results as we vary $\sigma_{\text {cut }}$ from $30 h \mathrm{Mpc}^{-1}$ to $60 h \mathrm{Mpc}^{-1}$. The strongest effect is on $G_{\Theta}$, and indeed inclusion of small scales noticeably lowers the measured $G_{\Theta}$. However, for all $\sigma_{\text {cut }} \geq 40 h \mathrm{Mpc}^{-1}$ the results have converged and the measured values are insensitive to the exact value of $\sigma_{\text {cut }}$. This indicates that the approximation for treating FoG should not be trusted for $\sigma<40 h \mathrm{Mpc}^{-1}$, while above this scale our approach is robust. This argument holds as well for $D_{A}$ and $H^{-1}$, though less extremely. Considerable caution should be applied to the use of the clustering data on small scales.

The outer contours provide another indicative behavior for $G_{\Theta}$. When $G_{\Theta}$ varies, the location of peaks on $2 \mathrm{D}$ BAO circle moves differently from the variation of $G_{b}$ [13]. The peak points run away from the pivot point (roughly where $\sigma \approx \pi$ ) as $G_{\Theta}$ decreases, and move toward it for increasing $G_{\Theta}$. From Fig. 3 we see that around the $\mathrm{BAO}$ ring the fourth and sixth contours recede away from the Planck LCDM prediction, while the fifth contour, which lies close to the pivot point, has not moved. This implies the measured $G_{\Theta}$ is smaller than the Planck LCDM prediction and indeed we find $G_{\Theta}=0.41 \pm 0.09$ in comparison with 0.46 predicted by Planck LCDM prediction. This is still within $68 \%$ CL however (note though that using a lower $\sigma_{\text {cut }}$ moves $G_{\Theta}$ to even smaller values).

The quantity $G_{b}$ represents the combination of density field and linear bias. As no cosmological model is assumed, the two are not separable. As shown in Table II, $G_{b}$ is measured to be $1.15 \pm 0.08$. If the Planck LCDM model is true, then the linear bias at $z=0.57$ can be estimated to be $b=2.0$ with $7 \%$ fractional error (recall that $\left.G_{b}=b D\right)$. This is consistent with what we observe from the simulation.

The velocity dispersion parameter $\sigma_{p}$ for FoG is measured to be $6.2_{-3.3}^{+3.6} h^{-1} \mathrm{Mpc}$. The FoG effect is at first order degenerate with $G_{\Theta}$; this causes weak constraints on both $\sigma_{p}$ and $G_{\Theta}$.

\section{B. Testing the cosmological framework}

Our model independent analysis allows several consistency tests.

1. In a Friedmann-Robertson-Walker (FRW) cosmology, $D_{A}$ is formed from an integral over $H^{-1}$.

2. Within general relativity and FRW, the growth rate $G_{\Theta}$ and expansion rate $H^{-1}$ are tied together.

3. Neutrino mass suppresses growth, so a measured consistency with Planck LCDM (i.e. minimal neutrino mass) disfavors higher neutrino mass (or requires a conspiracy with enhanced growth from modified gravity - but this would show up in the previous consistency test).

We therefore study the joint probability distribution between the measured cosmological quantities, e.g. the two dimensional likelihood contours of $G_{\Theta}$ vs $H^{-1}$. All such contours are marginalized over the remaining quantities.

In Fig. 5 we present two dimensional cosmological parameter contours in four different combinations. The x's represent the best values of Planck LCDM models; we see that our model independent analysis agrees within the $68 \%$ confidence level with the cosmology that assumed LCDM, general relativity, and minimal neutrino mass. 

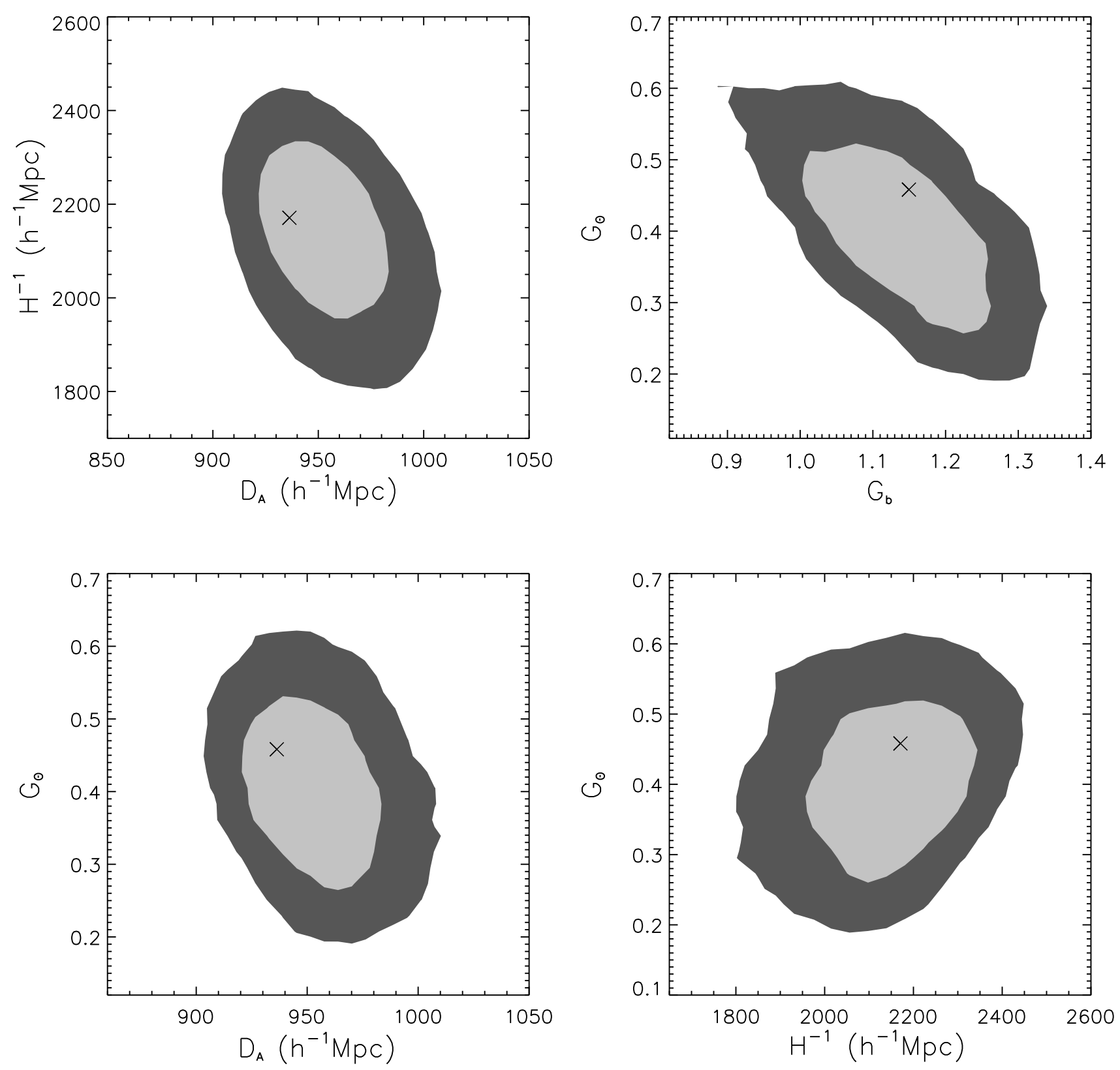

FIG. 5. The 2D joint likelihood contours at $68 \%$ and $95 \%$ CL measured for $D_{A}, H^{-1}, G_{b}$ and $G_{\Theta}$ are shown, using $s_{\text {cut }}=$ $50 \mathrm{~h} \mathrm{Mpc}^{-1}$ and $\sigma_{\text {cut }}=40 \mathrm{~h} \mathrm{Mpc}^{-1}$. The fiducial values in the Planck LCDM concordance model are shown by x's (see Table II).

This holds for all the measured distances and growth functions.

The first panel provides evidence for the FRW consistency relation of the background quantities of the distance and expansion rate. The second panel involves measurements of the perturbed quantities, from the density and velocity fields. As mentioned, $G_{b}$ is consistent with expectations for galaxy bias. The measured $G_{\Theta}$ can be converted to $f \sigma_{8}=0.43 \pm 0.09$, with the Planck LCDM model predicting $f \sigma_{8}=0.48$, again within $68 \%$
CL. (Note this would not hold if we naively included smaller scales where nonlinear modelling is not robust.)

The third and fourth panels, showing that the joint likelihoods for the background and growth quantities are consistent with Planck LCDM, can be thought of as a (weak, model independent) test of the general relativity criterion. That the fourth panel, showing the $G_{\Theta}-H^{-1}$ likelihood, is consistent with Planck LCDM (with minimal neutrino mass), also disfavors a larger neutrino mass and its accompanying suppression of growth. If one as- 


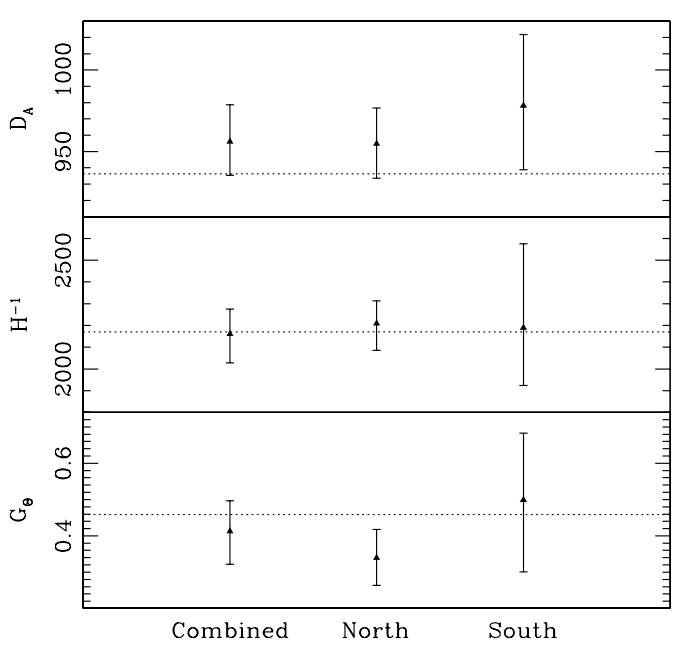

FIG. 6. The measured $D_{A}, H^{-1}$ and $G_{\Theta}$ are presented from the top to the bottom panels. Each one shows the results from the combined, northern, and southern skies, from left to right. The dotted lines represent the Planck LCDM predictions.

sumed that the background cosmology is truly the Planck LCDM model, then the growth measurement could be converted to an estimate of the gravitational growth index $\gamma$ [41] or a sum of neutrino masses $\sum m_{\nu}$. However for both of these the uncertainty on $G_{\Theta}$ is multiplied by a large prefactor so the constraints are weak.

\section{Comparison of North vs South}

Another interesting check involves a comparison of the estimated cosmological quantities using only the northern or southern hemisphere sky. The median RA and Dec of each patch is $(185,25)$ and $(2,10)$ respectively (see Fig. 1), so the centers of these two disjoint sky patches are separated by 145 degrees on the sky. The effective volume of the North is $4.5 \mathrm{Gpc}^{3}$ and that of the South is $1.5 \mathrm{Gpc}^{3}$.

Table II breaks down the cosmological results by hemisphere. All quantities are consistent within $68 \%$ CL; nevertheless, there are some interesting patterns worthwhile keeping an eye on as the data improves and the error bars shrink.

In the top and middle panels of Fig. 6 we present the measured $D_{A}$ and $H^{-1}$ for North and South separately, and the full survey combination. North and South agree with each other and with Planck LCDM predictions. The detailed numbers are shown in Table II. Note that the median measured $H^{-1}$ from the combined map does not lie between the North and South measured values; this occurs due to the non-Gaussian probability distribution for the measured $H^{-1}$ in the South - the mode value is $2116 h^{-1} \mathrm{Mpc}$.
For the measured coherent motion $G_{\Theta}$ in the bottom panel, the North is somewhat inconsistent $(\sim 1.5 \sigma)$ with the Planck LCDM prediction. From Table II, in the North $G_{\Theta}=0.34_{-0.08}^{+0.08}$ and in the South $G_{\Theta}=0.54_{-0.17}^{+0.16}$, while the LCDM fiducial has $G_{\Theta}=0.46$. The central values of North and South are noticeably different, though due to the large uncertainty from the small effective volume in the South this cannot be said to be statistically significant.

One might speculate about North-South anisotropy but this is disfavored due to the consistency of the measured $H^{-1}$ values. We have also checked that $z_{\text {eff }}$ is consistent between North and South, at the $0.05 \%$ level. Another possibility is inhomogeneity at the perturbation level, for example an anisotropic stress [42, 43]. With a quadrupole dependence, this would not have an effect if the North and South areas were $180^{\circ}$ apart, but could have a component as they are separated by $145^{\circ}$. Also note that the measured velocity dispersion $\sigma_{p}$ is different in North and South, which could support this. Alternately, the covariance between $\sigma_{p}$ and $G_{\Theta}$ is such that high $\sigma_{p}$ can damp the excess velocity growth of high $G_{\Theta}$, so that these (and the low $\sigma_{p}$, low $G_{\Theta}$ case for the North) lie along the degeneracy direction with LCDM.

\section{CONCLUSIONS}

We have carried out an analysis within a framework independent of the cosmological model, i.e. the specific energy density components such as dark energy or curvature. This uses the BOSS DR11 dataset that measures galaxy clustering over the largest volume yet surveyed with an effective redshift of $z=0.57$. We measure the angular distance $D_{A}$, expansion rate $H^{-1}$ (from the radial distance information), and velocity growth rate $G_{\Theta}$; all are consistent with the Planck LCDM prediction.

These are multiple, model independent tests of LCDM since the implications of each parameter is different. The measured $D_{A}$ is insensitive to uncertainties from contamination along the line of sight, but can be affected by the assumption of coherent (scale-independent) galaxy bias on the scales used, due to the degeneracy between $D_{A}$ and $G_{b}$. The value of the galaxy bias $b$ we derive is also consistent with other measurements. For accurate measurement of $H^{-1}$, the radial dependence of $\xi(\sigma, \pi)$ should be modelled robustly.

For $G_{\Theta}$, measured coherent motions are degenerate with the FoG effect which is problematic to model. If there is residual contamination from inaccurate modelling of the FoG effect, the coherent motions and hence velocity growth rate $G_{\Theta}$ or $f \sigma_{8}$ are underestimated. Since the theoretical model calibrated from simulations becomes increasingly inaccurate on small scales, we carefully examine the dependence of the results on the small scale cutoff in the measurements used. We find that convergence is achieved for $\sigma_{\text {cut }} \geq 40 h \mathrm{Mpc}^{-1}$, with bias arising if smaller scales are included - an important cau- 
tion. Our measurement corresponds to $f \sigma_{8}=0.43 \pm 0.09$, with the Planck LCDM prediction of $f \sigma_{8}=0.48$.

Considering the joint likelihood of these cosmological quantities, we find consistency with the Planck LCDM model at $68 \%$ CL. By comparing the quantities to each other, we can make three general consistency tests of the cosmological framework. We check consistency with the FRW framework, with general relativity, and with minimal neutrino mass and find that all are within $68 \%$ CL.

Comparing the galaxy measurements from the North and South sky samples separately, we continue to find consistency with Planck LCDM. Here the reduced effective volume makes the error bars larger, but there are slight discrepancies worth testing with future data from larger surveys such as DESI or LSST. For example, the growth rate in the North has $G_{\Theta}=0.34(\sim 1.5 \sigma$ from Planck LCDM) while the South has $G_{\Theta}=0.54$ (though again the error bar is so large that these are consistent within $68 \% \mathrm{CL}$ ). One speculative explanation is the presence of anisotropic stress (affecting the perturbations while keeping the background quantities consistent), but covariance between $G_{\Theta}$ and $\sigma_{p}$ (also measured to be different in North and South) is another possibility. Again, upcoming larger sky surveys will be valuable in testing cosmology in different directions.

\section{ACKNOWLEDGMENTS}

Numerical calculations were performed by using a high performance computing cluster in the Korea Astronomy and Space Science Institute and we also thank the Korea Institute for Advanced Study for providing computing resources (KIAS Center for Advanced Computation Linux Cluster System). EL was supported in part by US DOE grant DE-SC-0007867 and Contract No. DEAC02-05CH11231. We thank Marc Manera for providing the mock simulations and Shinji Mukohyama for helpful comments on anisotropic stress.

Funding for SDSS-III has been provided by the Alfred P. Sloan Foundation, the Participating Institutions, the National Science Foundation, and the U.S. Department of Energy Office of Science. The SDSS-III web site is http://www.sdss3.org/.

SDSS-III is managed by the Astrophysical Research Consortium for the Participating Institutions of the SDSS-III Collaboration including the University of Arizona, the Brazilian Participation Group, Brookhaven National Laboratory, Carnegie Mellon University, University of Florida, the French Participation Group, the German Participation Group, Harvard University, the Instituto de Astrofisica de Canarias, the Michigan State/Notre Dame/JINA Participation Group, Johns Hopkins University, Lawrence Berkeley National Laboratory, Max Planck Institute for Astrophysics, Max Planck Institute for Extraterrestrial Physics, New Mexico State University, New York University, Ohio State University, Pennsylvania State University, University of Portsmouth, Princeton University, the Spanish Participation Group, University of Tokyo, University of Utah, Vanderbilt University, University of Virginia, University of Washington, and Yale University.
[1] W. E. Ballinger, J. A. Peacock, A. F. Heavens 1996, MNRAS, 282, 877

[2] T. Matsubara, \& Y. Suto 1996, ApJL, 470, 1

[3] N. Padmanabhan and M. J. White, 1, Phys. Rev. D 77, 123540 (2008)

[4] W. Hu and Z. Haiman, Phys. Rev. D 68, 063004 (2003)

[5] E. Gaztanaga, A. Cabre and L. Hui, Mon. Not. Roy. Astron. Soc. 399, 1663 (2009)

[6] B. A. Reid, L. Samushia, M. White, W. J. Percival, M. Manera, N. Padmanabhan, A. J. Ross and A. G. Sanchez et al., Mon. Not. Roy. Astron. Soc. 426, 2719 (2012)

[7] L. Samushia, B. A. Reid, M. White, et al. 2014, MNRAS, 439, 3504

[8] F. Beutler, S. Saito, H.-J. Seo, et al. 2013, arXiv:1312.4611

[9] T. Okumura, T. Matsubara, D. J. Eisenstein, I. Kayo, C. Hikage, A. S. Szalay, \& D. P. Schneider (2008) Astrophys. J., 676,889

[10] A. G. Sanchez, F. Montesano, E. A. Kazin, et al. 2014, MNRAS, 440, 2692

[11] C. Alcock and B. Paczynski, Nature 281, 358 (1979).

[12] M. López-Corredoira 2014, Astrophys. J.781, 96

[13] Y. -S. Song, T. Okumura and A. Taruya, Phys. Rev. D
89, 103541 (2014) [arXiv:1309.1162 [astro-ph.CO]].

[14] E. V. Linder, M. Oh, T. Okumura, C. G. Sabiu and Y. -S. Song, Phys. Rev. D 89, 063525 (2014) [arXiv:1311.5226 [astro-ph.CO]].

[15] N. Kaiser, Mon. Not. Roy. Astron. Soc. 227, 1 (1987).

[16] A. J. S. Hamilton 1998, ASSL, 231, 185

[17] J. C. Jackson, 1972, MNRAS156, 1

[18] R. Scoccimarro, Astrophys. J. 517, 531 (1999)

[19] R. Scoccimarro, Phys. Rev. D 70, 083007 (2004)

[20] A. Taruya, T. Nishimichi and S. Saito, Phys. Rev. D 82 , $063522(2010)$

[21] A. Taruya and T. Hiramatsu, Astrophys. J. 674, 617 (2008)

[22] A. Taruya, F. Bernardeau, T. Nishimichi and S. Codis, Phys. Rev. D 86, 103528 (2012)

[23] Y. -S. Song and W. J. Percival, JCAP 0910, 004 (2009)

[24] M. Manera, R. Scoccimarro, W. J. Percival, L. Samushia, C. K. McBride, A. Ross, R. Sheth and M. White et al., Mon. Not. Roy. Astron. Soc. 428, no. 2, 1036 (2012)

[25] L. Anderson, E. Aubourg, S. Bailey, et al. 2012, MNRAS, 427,3435

[26] D. Schlegel, M. White, \& D. Eisenstein, arXiv:0902.4680

[27] D. J. Eisenstein, D. H. Weinberg, Agol, E., et al. 2011, AJL, 142, 72 
[28] A. S. Bolton, D. J. Schlegel, É Aubourg, et al. 2012, AJL, 144,144

[29] K. S. Dawson, D. J. Schlegel, C. P. Ahn, et al. 2013, AJL, 145,10

[30] S. A. Smee, J. E. Gunn, A. Uomoto, et al. 2013, AJL, 146,32

[31] D. G. York, J. Adelman, J. E. Anderson Jr., et al. 2000, AJL, 120, 1579

[32] J. E. Gunn, W. A. Siegmund, E. J. Mannery, et al. 2006, AJL, 131, 2332

[33] The data and random catalogues will be available online at http://data.sdss3.org/sas/dr11/boss/

[34] S. E. Nuza, A. G. Sánchez, F. Prada, et al. 2013, MNRAS, 432, 743

[35] H. A. Feldman, N. Kaiser, \& J. A. Peacock, 1994, Astrophys. J., 426, 23
[36] A. J. Ross, W. J. Percival, A. G. Sánchez, et al. 2012, MNRAS, 424, 564

[37] T. Okumura, U. Seljak, \& V. Desjacques (2012) J. Cosmology Astropart. Phys., 11, 014 [arXiv:1206.4070]

[38] S. D. Landy, \& A. S. Szalay, 1993, Astrophys. J., 412, 64

[39] L. Anderson, É. Aubourg, S. Bailey, et al. 2014, MNRAS, 441, 24

[40] Y. -S. Song, C. G. Sabiu, I. Kayo and R. C. Nichol, JCAP 1105, $020(2011)$

[41] E. V. Linder, 2005, Phys. Rev. D, 72, 043529

[42] A. De Felice, A. E. Gumrukcuoglu, C. Lin and S. Mukohyama, Class. Quant. Grav. 30, 184004 (2013) [arXiv:1304.0484 [hep-th]].

[43] A. E. Gumrukcuoglu, C. Lin and S. Mukohyama, Mod. Phys. Lett. A 28, 1340016 (2013). 